\title{
Influencia de la intensidad de clara y tipo de dosel en el ciclo interno de nutrientes y el crecimiento secundario de pino silvestre en un bosque mixto de los Pirineos occidentales
}

\author{
I. Primicia $1,2, *$ \\ (1) Departamento de Ciencias del Medio Natural, Universidad Pública de Navarra, Campus de Arrosadía s/n, Pamplona, 31006 Navarra, Spain. \\ (2) Present address: Department of Silviculture, Faculty of Forestry and Wood Sciences, Czech University of Life Sciences- Prague, Kamýcka 129, 16521 \\ Praha 6 Suchdol, Czech Republic \\ * Autor de correpondencia: I. Primicia [irantzu.primicia@unavarra.es]
}

> Recibido el 31 de octubre de 2013, aceptado el 15 de diciembre de 2013.

Primicia, I. 2013. Influencia de la intensidad de clara y tipo de dosel en el ciclo interno de nutrientes y el crecimiento secundario de pino silvestre en un bosque mixto de los Pirineos occidentales. Ecosistemas 22(3):131-135. Doi.: 10.7818/ECOS.2013.22-3.19

Debido a sus múltiples beneficios (ej. mayor diversidad, protección y resistencia frente a enfermedades, estrés abiótico o perturbaciones), se ha recomendado la conversión de bosques puros de coníferas en bosques mixtos en zonas potencialmente sensibles a cambios climáticos y medioambientales (Spiecker 2003). El mantenimiento y mejora de las masas mixtas se obtienen frecuentemente a través de tratamientos selvícolas como las claras (Spiecker 2003). Por ello, un conocimiento sólido sobre el funcionamiento del ecosistema en bosques mixtos gestionados resulta imprescindible. En este trabajo se analiza la influencia de la intensidad de clara a medio plazo (8-9 años tras la clara) y el tipo de dosel sobre el crecimiento secundario de pino silvestre y el ciclo de nutrientes (cambios químicos en suelo, retranslocación, y trascolación). El estudio forma parte de una investigación a largo plazo sobre el funcionamiento del bosque y la sostenibilidad de las claras forestales desde una perspectiva ecológica. El área de estudio está localizada en Aspurz, Navarra, en los Pirineos occidentales. La precipitación y temperatura media anual es de $913 \mathrm{~mm}$ y $12.0^{\circ} \mathrm{C}$ respectivamente. Se trata de una masa regular dominada por pino silvestre (Pinus sylvestris L.), donde la cobertura del haya (Fagus sylvatica L.) se está expandiendo en relación a la del pino debido a su carácter tolerante. El diseño experimental está constituido por nueve parcelas donde se aplicaron tres intensidades de clara en 1999 (extracción del $0 \%, 20 \%$ y $30 \%$ del área basimétrica de pino silvestre, $n=3$ parcelas por intensidad de clara). Cada parcela está dividida en dos subparcelas irregulares: dosel puro pino (DP) y dosel mixto pino-haya (DM). La relación de variables analizadas en el estudio y una breve descripción de los métodos empleados pueden encontrarse en la Tabla 1.

Las claras causaron un efecto positivo débil y temporal (en 2002) sobre el crecimiento de pino silvestre (Primicia et al. 2013a, Fig. 1a). Además, se observó una reducción gradual del crecimiento de pino silvestre en el DM en las parcelas control a medida que el haya se fue desarrollando y ocupando los estratos arbóreos superiores (Fig. 1b). Estos resultados fueron corroborados a nivel intra-anual por un frecuente mayor incremento radial y número de traqueidas en el DP respecto al DM en las parcelas control. Estos patrones se explicaron por diferencias en el nivel de competencia
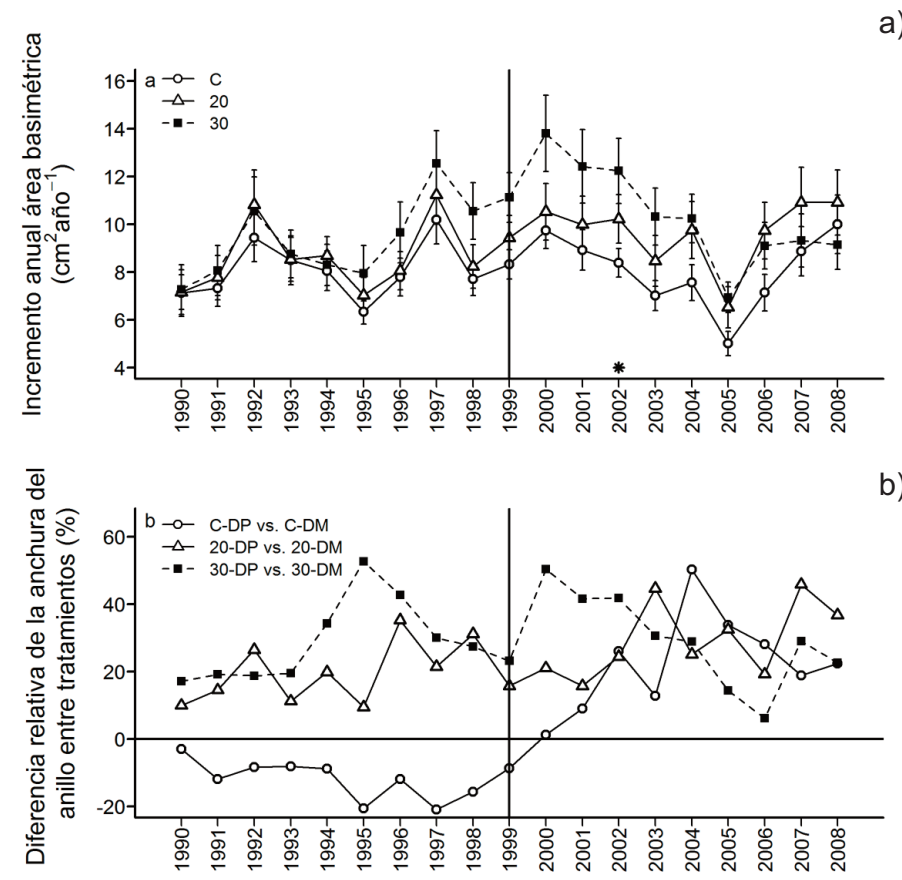

Figura 1. Incremento anual del área basimétrica (medias $\pm E E$ ) para cada intensidad de clara (a) y diferencia relativa de la anchura del anillo $(A A)$ entre C-DM vs. C-DP, 20-DM vs. 20-DP y 30-DM vs. 30-DP (b). Los cálculos, por

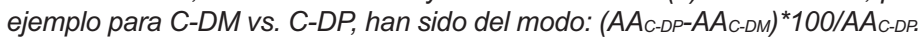
Fuente: Primicia et al. (2013a, modificado). Abreviaciones: C, parcelas control; 20, parcelas con clara ligera (20\%); 30, parcelas con clara moderada (30\%); DM, dosel mixto de pino y haya; DP, dosel puro de pino; C-DM, dosel mixto en parcelas control; C-DP, dosel puro en parcelas control; 20-DM, dosel mixto en parcelas con clara ligera (20\%); 20-DP, dosel puro en parcelas con clara ligera (20\%); 30-DM, dosel mixto en parcelas con clara moderada (30\%); 30-DP, dosel puro en parcelas con clara moderada (30\%). Niveles de significación: * $P<0.05$; ${ }^{* *} P<0.01$; ${ }^{* *} P<0.001$. Las diferencias significativas para el incremento del área basimétrica están indicadas arriba, y para la anchura del anillo, abajo. La línea vertical indica el año cuando se ejecutó la clara. 
Tabla 1. Relación de variables medidas en el estudio y breve descripción de los métodos empleados

\begin{tabular}{cccc}
\hline $\begin{array}{c}\text { Grupo } \\
\text { de variables }\end{array}$ & Periodo de estudio & Material y métodos & Variables \\
\hline
\end{tabular}

\begin{tabular}{cc}
\hline Dasométricas & $1999 ; 2008 \quad \begin{array}{l}\text { Conteo, georreferenciación y medición con forcípula } \\
\text { de todos los árboles incluidos en las parcelas }\end{array}$ \\
$2008 \quad \begin{array}{l}\text { Medición con hipsómetro vertex de árboles } \\
\text { seleccionados para el estudio del crecimiento de } \\
\text { pino silvestre }\end{array}$ \\
\end{tabular}

\begin{tabular}{ll}
\hline Microclima & Junio 2007- \\
& Marzo 2009
\end{tabular}

Registro horario en data-loggers Hobo. Para su relación con el crecimiento intra-anual de pino silvestre se calcularon valores medios, mínimos y máximos de los diez días previos a la medición de dendrómetros o toma de muestras xilogenéticas

Densidad de árboles por parcela, diámetro normal, área basimétrica

Altura, longitud de copa viva

Cálculo de índices de competencia de los árboles vecinos

Humedad y temperatura ambiental

Humedad y temperatura del suelo

Radiación fotosintéticamente activa (PAR)

\begin{tabular}{lcl}
\hline $\begin{array}{l}\text { Crecimiento } \\
\text { secundario } \\
\text { de pino silvestre }\end{array}$ & $1990-2008$ & $\begin{array}{l}\text { Datación y medición de cores recogidos en marzo } \\
2009\end{array}$ \\
& 2008 & $\begin{array}{l}\text { Medición (quincenal/ mensual) de dendrómetros de } \\
\text { banda }\end{array}$
\end{tabular}

Anchura del anillo, incremento del área basimétrica

banda

Incremento radial acumulado, tasas de incremento radial

Análisis histológico de muestras xilogenéticas recogidas con minibarrena Trephor (Mayo, Junio, Julio, Septiembre)

Número de células del cámbium, número de traqueidas en fase de expansión radial, en fase de engrosamiento de la pared celular, número total de traqueidas, tasa de producción de traqueidas

\begin{tabular}{lll}
\hline $\begin{array}{l}\text { Contenido de } \\
\text { nutrientes } \\
\text { en el suelo }\end{array}$ & Marzo 2007- & $\begin{array}{l}\text { Muestras de la capa mineral superficial del suelo } \\
(12 \mathrm{~cm} \text { de profundidad) recogidas en puntos } \\
\text { seleccionados al azar. }\end{array}$
\end{tabular}

Contenido de $\mathrm{NH}_{4}{ }^{+}-\mathrm{N}$ y $\mathrm{NO}_{3}{ }^{-} \mathrm{N}$ (mensual) y $\mathrm{P}$

disponible (bimensual)

Incubación de muestras en campo (mensual)

Tasa neta de mineralización de $\mathrm{NH}_{4}{ }^{+}-\mathrm{N}$ y $\mathrm{NO}_{3}{ }^{-}-\mathrm{N}$ (mensual)

\begin{tabular}{llll}
\hline $\begin{array}{l}\text { Contenido de } \\
\text { nutrientes } \\
\text { y retranslocación } \\
\text { en acículas } \\
\text { de pino silvestre }\end{array}$ & $\begin{array}{l}\text { Marzo 2007- } \\
\text { Febrero 2009 }\end{array}$ & $\begin{array}{l}\text { Muestras recogidas de la parte superior de las } \\
\text { copas (bimensual) }\end{array}$ & $\begin{array}{l}\text { Longitud, peso y contenido de N, P y K de } \\
\text { acículas en verde }\end{array}$ \\
\hline $\begin{array}{l}\text { Agua de } \\
\text { trascolación }\end{array}$ & $\begin{array}{l}\text { Marzo 2007- } \\
\text { Febrero 2009 }\end{array}$ & $\begin{array}{l}\text { Muestras de precipitación recogidas en tres claros desfronde (mensual) } \\
\text { situados cerca de las parcelas (mensual) }\end{array}$ & $\begin{array}{l}\text { Longitud, peso y contenido de N, P y K de } \\
\text { acículas de desfronde }\end{array}$ \\
& & $\begin{array}{l}\text { Muestras de trascolación (bajo el dosel arbóreo, } \\
\text { prensual) }\end{array}$ & $\begin{array}{l}\text { Volumen y composión química del agua de } \\
\text { trascolación }\end{array}$ \\
& & muímica del agua de
\end{tabular}

de árboles vecinos, y en el tamaño (altura, altura de copa) y estado hídrico del árbol (humedad relativa del aire, humedad del suelo, trascolación), causadas por los diferentes tratamientos. De hecho, el estado hídrico del árbol fue uno de los principales factores limitantes del crecimiento de pino silvestre, resaltando el carácter mediterráneo del área de estudio (Fig. 2).

El crecimiento secundario arbóreo puede estar también influenciado por la disponibilidad de nutrientes. La clara ligera (20\%) produjo una reducción consistente del contenido de $\mathrm{NO}_{3}-\mathrm{N}$ y un descenso temporal del contenido de $\mathrm{NH}_{4}{ }^{+}-\mathrm{N}$, que pudo estar causado por diferencias en la absorción de $\mathrm{N}$ por los árboles en las parcelas con diferente intensidad de clara. El contenido de $\mathrm{N}$ inorgánico fue mayor en el suelo de DP incluso cuando la mineralización neta de $\mathrm{N}$ fue similar entre ambos tipos de doseles, sugiriendo que el haya tiene una mayor capacidad de absorción de $\mathrm{N}$ que el pino silvestre. El crecimiento secundario de pino silvestre estuvo relacionado (positivamente) con la tasa neta de amonificación media durante el periodo de crecimiento en 2007. La ausencia de dicha relación en 2008 pone de manifiesto la complejidad de los procesos integrantes del ciclo de nutrientes y su relación con el crecimiento arbóreo, así como la importancia de las variaciones inter-anuales. Además, el ratio de $\mathrm{N}$ : $\mathrm{P}$ en las acículas de pino y la inmovilización de $\mathrm{P}$ durante la descomposición de hojarasca en las mismas parcelas de estudio (Blanco et al. 2011) sugieren que los pinos de este bosque pueden estar también limitados por P. La ausencia de un efecto significativo del P dis- ponible en el suelo sobre el crecimiento de pino silvestre pudo haberse debido, por ejemplo, a un cierto desfase entre el crecimiento arbóreo y la adquisición de nutrientes del suelo.

La eficiencia de retranslocación de los nutrientes fue estimada antes y durante la senescencia en las acículas de pino de diferentes cohortes (Fig. 3). El P fue el nutriente con mayores tasas de retranslocación, seguido por el $\mathrm{N}$ y finalmente, por el K (Primicia et al. 2013b). Las claras afectaron al contenido de N y P en acículas de cohortes viejas, pero también a la longitud, peso y contenido de $\mathrm{K}$ de acículas jóvenes y viejas, indicando que algunos efectos de las mismas permanecieron incluso 8-9 años tras su ejecución. Generalmente, las acículas fueron más ligeras, cortas y con menor contenido de nutrientes en las parcelas con clara moderada (30\%), aparentemente debido a diferencias en el estado hídrico de las acículas. Se observó un mayor efecto del tipo de dosel sobre las características de las acículas de cohortes nuevas, aunque, estos efectos dependieron del año de estudio. Una mayor retranslocación de nutrientes en las acículas de pino silvestre sería esperable en el DM para contrarrestar la competencia del haya sobre el pino. Sorprendentemente, la eficiencia de retranslocación de nutrientes fue mayor (aunque no significativamente) en el DP que en el DM (Fig. 4), lo que podría estar relacionado con el mayor crecimiento secundario de pino silvestre en el DP. No obstante, no se encontró ninguna relación significativa entre el crecimiento de pino silvestre y la eficiencia de retranslocación de nutrientes. 


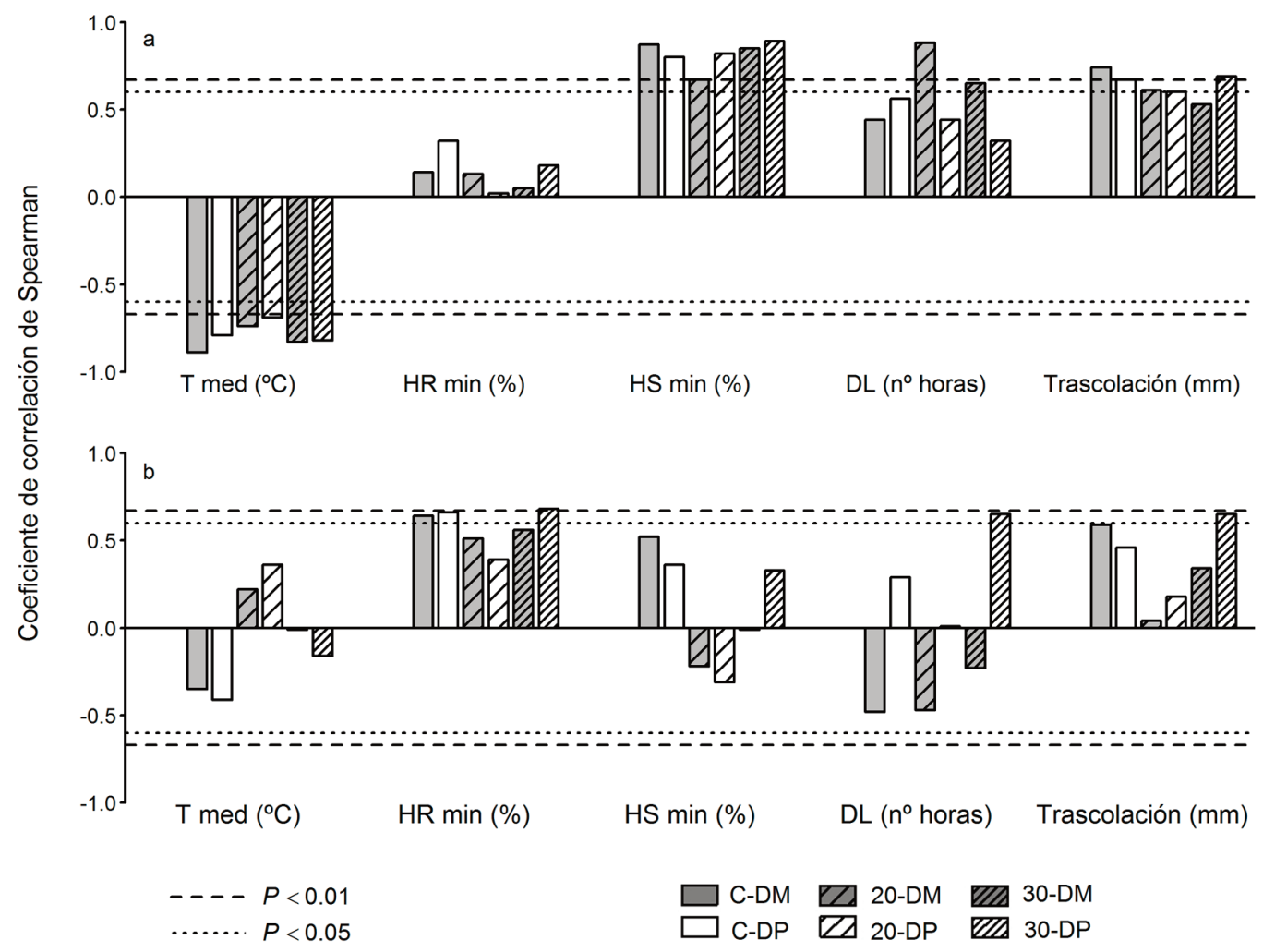

Figura 2. Coeficientes de correlación de Spearman entre variables microclimáticas (medias, mínimas y máximas calculadas para un periodo de 10 días previo al muestreo) y el número traqueidas en fase de expansión radial (a) y la tasa de producción de traqueidas (b) en pino silvestre en los diferentes tratamientos. Fuente: Primicia et al. (2013a, modificado). Abreviaciones: T med, Temperatura del aire media; HR min, humedad relativa mínima; HS min, humedad del suelo mínima; LD, longitud del día. Las abreviaciones de los tratamientos son como en la Fig. 1. Niveles de significación: $P$ < 0.05 (línea punteada); $P<0.01$ (línea discontinua).
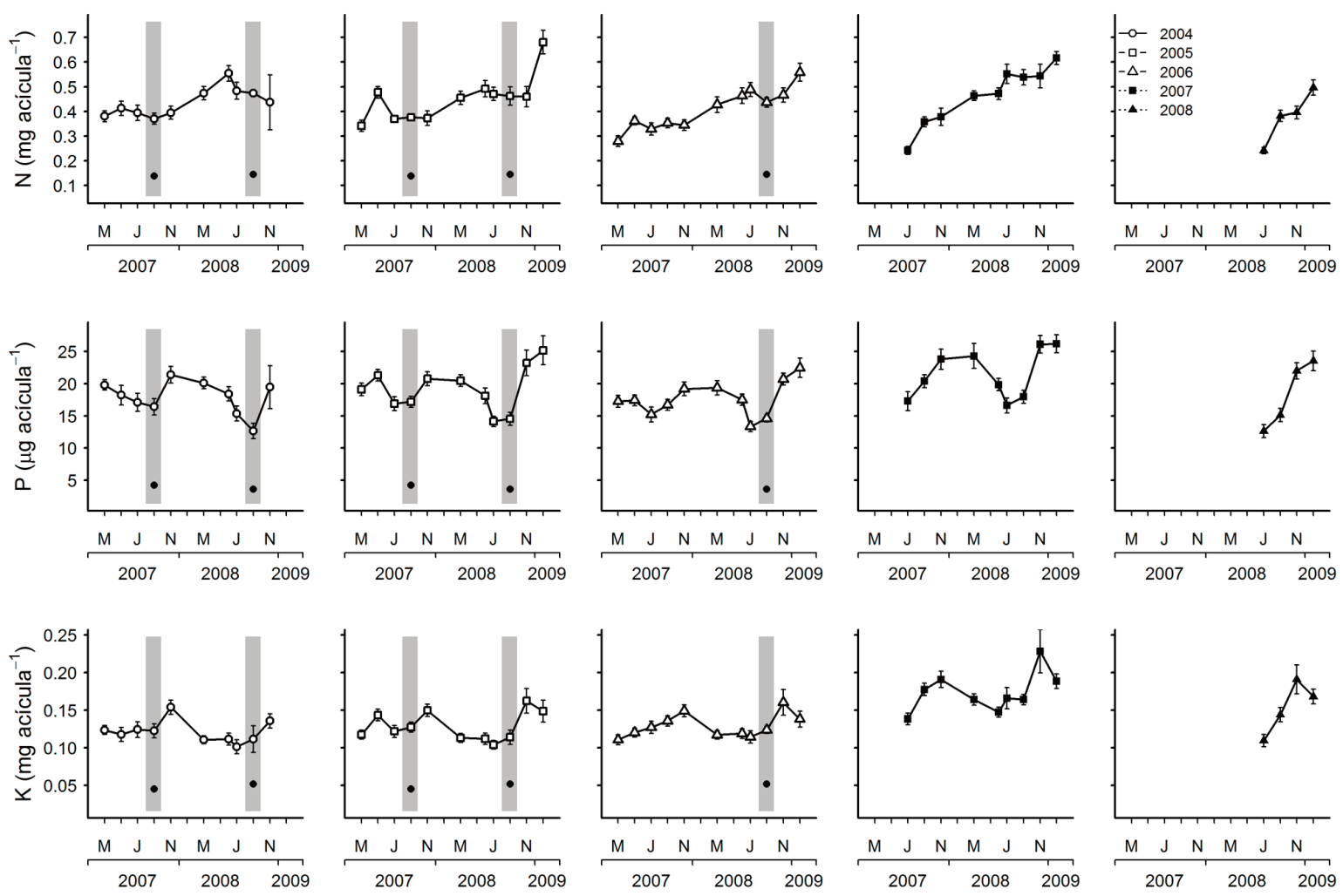

Figura 3. Contenido de nutrientes (N, Py K, medias \pm EE) en acículas de pino silvestre de diferentes cohortes. Fuente: Primicia et al. (2013b). Las barras grises indican el periodo principal de senescencia anual para las cohortes consideradas como más susceptible de abscisión. Los puntos negros indican el contenido de nutrientes medio en acículas del desfronde durante el pico anual de desfronde de acículas. La eficiencia en la retranslocación previo a la abscisión de acículas en las diferentes cohortes (excepto cohorte 2008) fue estimada como: $E R_{V}=\left(\max -N_{V}-N_{s}\right) / \max -N_{v}$, siendo max- $N_{V}$ el contenido de nutriente en acículas en verde durante el mes con máxima concentración de nutriente y $N_{s}$ el contenido de nutriente en acículas pre-senescentes durante el mes con mayor desfronde de acículas (Agosto-Septiembre 2007; Septiembre-Octubre 2008). La eficiencia de retranslocación durante la senescencia $(E R S)$ fue estimado durante el pico anual de desfonde de acículas como $E R S=\left(N_{S}-N_{D}\right) / N_{S}$, donde $N_{D}$ se refiere al contenido de nutrientes en acículas de desfronde 

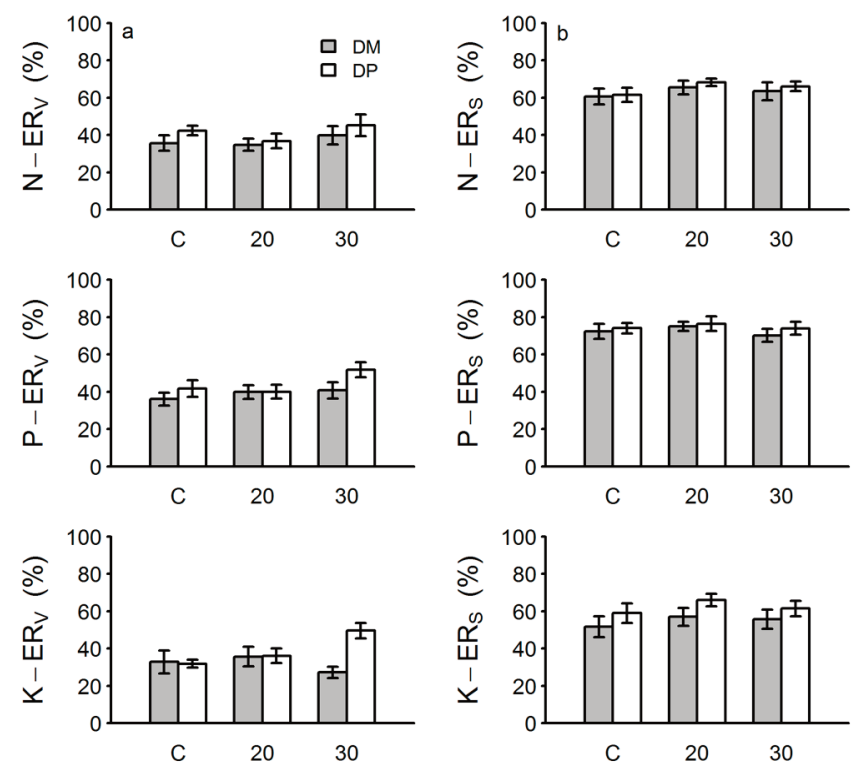

Figura 4. Eficiencia de retranslocación (medias $\pm E E$ ) previo a la abscisión $(E R v$, a) y durante la senescencia (ERs, b) de $N, P$ y $K$ en acículas de pino silvestre en los diferentes tratamientos. Fuente: Primicia et al. (2013b). Las abreviaciones de los tratamientos son como en la Fig. 1.
La partición de la precipitación y los flujos de nutrientes en el agua de trascolación se estudiaron a partir del muestreo mensual de precipitación y trascolación. Durante la estación de crecimiento se observó un incremento en la absorción de nutrientes por el dosel arbóreo a través de deposición húmeda (principalmente $\mathrm{NH}_{4}{ }^{+}-\mathrm{N}$ y $\mathrm{PO}_{4}{ }^{3-} \mathrm{P}$, y $\mathrm{NO}_{3}-\mathrm{N}$ en primavera), posiblemente para cubrir el incremento de la demanda de nutrientes por los árboles. Aunque la intensidad de clara únicamente afectó a las características del agua de trascolación ocasionalmente, éstas estuvieron altamente influenciadas por el tipo de dosel. El efecto del tipo de dosel sobre el agua de trascolación pudo estar relacionado con diferencias entre el pino silvestre y el haya en la fisiología y permeabilidad del follaje y en la estructura de la masa forestal. Por ejemplo, el volumen de agua de trascolación fue siempre menor, y la deposición de $\mathrm{NO}_{3}{ }^{-} \mathrm{N}$ en la misma mayor, en el DM, probablemente relacionado con la superposición de los doseles de pino silvestre (estrato dominante) y haya (estrato dominado). Como resultado, el DM dispone de una mayor capa filtradora y, por tanto, mayor interceptación de precipitación y deposición seca. Sin embargo, el efectodel tipo de dosel sobre las características del agua de trascolación varió frecuentemente a lo largo del periodo de estudio, destacando la complejidad de estos procesos y su repuesta a la gestión forestal.

En resumen, en el DM, en comparación con el DP, se observó un incremento de la competencia árbol-a-árbol sobre el pino silvestre y una reducción de la humedad del suelo, que estuvieron relacionados con un descenso en el crecimiento de pino silvestre (Fig. 5a).
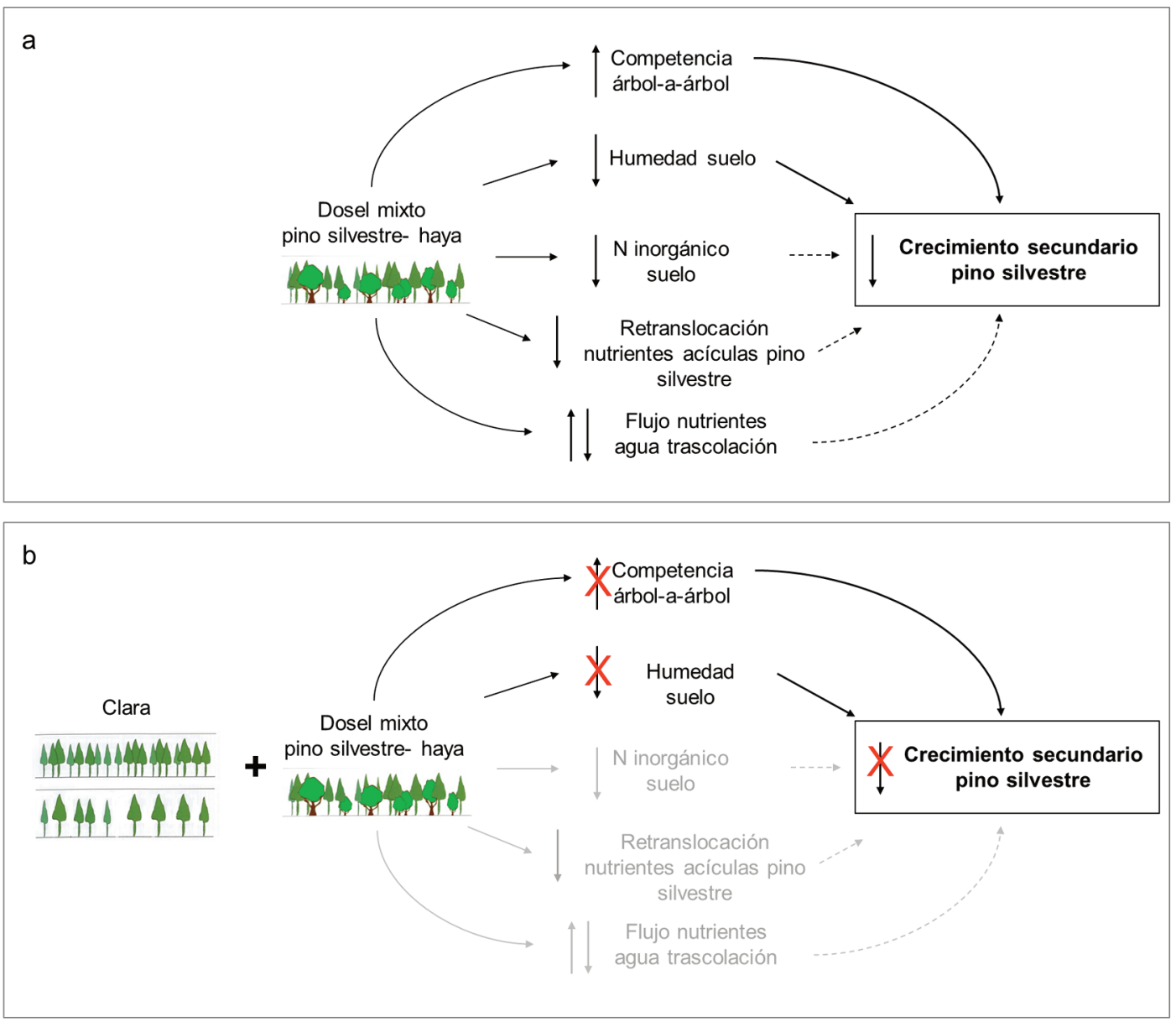

Figura 5. Modelo conceptual del efecto de los tratamientos sobre las variables estudiadas. a). La presencia del haya en el dosel mixto pino silvestre- haya provocó, en comparación con el dosel puro, un aumento en la competencia árbol-a-árbol sobre el pino silvestre y una disminución de la humedad del suelo. Estos efectos estuvieron relacionados con un descenso en el crecimiento secundario de pino silvestre en el dosel mixto en relación al dosel puro. En el dosel mixto también se observó una disminución del $N$ inorgánico del suelo y de la retranslocación de nutrientes ( $N$, $P$, K) en las acículas de pino silvestre, y diferentes efectos sobre el flujo de nutrientes en el agua de trascolación. Estos procesos podrían estar relacionados con el descenso de crecimiento de pino silvestre en el dosel mixto, pero no se encontraron relaciones significativas entre dichas variables. b). En las parcelas aclaradas, el efecto de la presencia del haya sobre la competencia árbol-a-árbol sobre el pino silvestre y la humedad del suelo, variables relacionadas con el crecimiento de pino silvestre en el área de estudio, desapareció. Como resultado, el crecimiento de pino silvestre fue similar en las manchas mixtas que en las manchas puras de pino en las parcelas aclaradas. Líneas continuas representan relaciones significativas y líneas discontinuas, relaciones hipotéticas. 
También se observó una disminución del contenido de $\mathrm{N}$ inorgánico en el suelo y en la retranslocación de nutrientes en las acículas de pino, además de una alteración en las características del agua de trascolación en el DM respecto al DP. Estos cambios en el ciclo de nutrientes debido a la mezcla de especies arbóreas pudo haber estado relacionada con la reducción del crecimiento del pino silvestre en el DM, pero no se encontró ninguna relación significativa entre dichas variables. Por tanto, aparentemente, en las parcelas aclaradas el crecimiento de pino silvestre fue similar en ambos tipos de manchas debido a la inexistencia de ese aumento en la competencia y reducción en la humedad del suelo en el DM comparado con el DP (Fig. 5b). En la zona de estudio, futuras intervenciones (claras) pueden amplificar las diferencias en la respuesta a la intensidad de clara, mientras que el efecto del tipo de dosel probablemente aumentará a medida que el haya vaya desarrollándose y alcanzando los estratos superiores de la masa forestal. Sin embargo, la respuesta del ciclo de nutrientes y el crecimiento secundario arbóreo a este desarrollo del bosque es incierta. Nuestros resultados resaltan la necesidad de realizar estudios a largo plazo sobre estos procesos para alcanzar una gestión forestal sostenible en esta zona climática transicional, vulnerable a cambios globales.

\section{Agradecimientos}

Esta tesis doctoral ha sido desarrollada dentro del marco del proyecto AGL2006-08288 (Ministerio de Economía y Competitividad, MINECO, del Gobierno de España). I. Primicia ha sido financiada por el programa FPI del MINECO y el Fondo Social Europeo (FSE).

\section{Referencias}

Blanco, J.A., Imbert, J.B., Castillo, F.J. 2011. Thinning affects Pinus sylvestris needle decomposition rates and chemistry differently depending on site conditions. Biogeochemistry 106:397-414.

Primicia, I., Camarero, J.J., Imbert, J.B., Castillo, F.J. 2013a. Effects of thinning and canopy type on growth dynamics of Pinus sylvestris: inter-annual variations and intra-annual interactions with microclimate. European Journal of Forest Research 132: 121-135.

Primicia, I., Imbert, J.B., Traver, M.C., Castillo, F.J. 2013b. Inter-specific competition and management modify the morphology, nutrient content and resorption in Scots pine needles. European Journal of Forest Research. DOI: 10.1007/s10342-013-0753-7

Spiecker, H. 2003. Silvicultural management in maintaining biodiversity and resistance of forests in Europe-temperate zone. Journal of Environmental Management 67:55-65.

\section{IRANTZU PRIMICIA ÁLVAREZ}

Influence of thinning and canopy type on the internal nutrient cycling and the secondary growth of Pinus sylvestris L. in a mixed forest in the Pyrenees.

\section{Tesis Doctoral}

Universidad Pública de Navarra

Noviembre de 2012

Dirección: Drs. J. Bosco Imbert y Federico J. Castillo

\section{Publicaciones resultantes de la tesis}

Primicia, I., Camarero, J.J., Imbert, J.B., Castillo, F.J. 2013. Effects of thinning and canopy type on growth dynamics of Pinus sylvestris: interannual variations and intra-annual interactions with microclimate. European Journal of Forest Research 132:121-135.

Primicia, I., Imbert, J.B., Traver, M.C., Castillo, F.J. 2013. Inter-specific competition and management modify the morphology, nutrient content and resorption in Scots pine needles. European Journal of Forest Research DOI: 10.1007/s10342-013-0753-7

Primicia, I., Arzoz, I., Arricibita, F.J., Imbert, J.B., Unzu, M., García, S., Castillo, F.J. 2008. Influence of thinning and type of canopy on soil physicochemical characteristics in a mixed forest from the western Pyrenees. In: Stadler, J.,Schöppe, F., Frenzel, M. (eds.). Proceedings of the EURECO-GFOE 2008 "Biodiversity in an Ecosystem Context". 15-19 Septiembre 2008, Leipzig, Germany. EURECO. Leipzig, Germany.

Primicia, I., Camarero, J.J., Imbert, J.B., Castillo, F.J. 2009. Influencia de la intensidad de clara y el tipo de dosel sobre la dinámica del incremento radial y la xilogénesis de Pinus sylvestris L. En: Libro de actas del IX Congreso Nacional de la AEET. 18-22 Octubre 2009, Úbeda, Jaén, España. AEET. Madrid, España.

Primicia, I., Esnaola, M., Arricibita, F.J., Imbert, J.B., Unzu, M., García, S., Castillo, F.J. 2011. Soil physicochemical characteristics are affected by thinning and type of canopy in a Pyrenean mixed forest. In: Abstract book of the 12th European Ecological Federation (EFF) Congress, 25- 29 Septiembre 2011, Ávila, Spain. AEET. Madrid, Spain.

Primicia, I., Imbert, J.B., Unzu, M., García, S., Castillo, F.J. 2011. Influence of thinning and type of canopy on volume and chemical composition of throughfall in a mixed forest from the western Pyrenees. In: Abstract book of the Environment, Pyrenees International Conference, 19-21 Octubre 2011, Pamplona, Spain. University of Navarra. Pamplona. Spain.

Primicia, I., Imbert, J.B., Traver, M.C., Castillo, F.J. 2013. La competencia inter-específica y la gestión forestal modifican la retranslocación de nutrientes en acículas de pino silvestre. En: Libro de actas del XI Congreso Nacional de la AEET. 6-10 Mayo 2013, Pamplona, Navarra. AEET. Madrid, España. 